\title{
Numerical investigation on the forming behaviour of stainless/carbon steel bimetal composite
}

\author{
Zhou $\mathrm{Li}^{1}$, Jingwei Zhao ${ }^{1}$, Qingfeng Zhang ${ }^{2}$, Sihai Jiao ${ }^{2}$, and Zhengyi Jiang ${ }^{1, *}$ \\ ${ }^{1}$ School of Mechanical, Materials, Mechatronic and Biomedical Engineering, University of \\ Wollongong, Wollongong NSW 2522, Australia \\ ${ }^{2}$ Baosteel Research Institute (R\&D Centre), Baoshan Iron \& Steel Co., Ltd., Shanghai 200431, China
}

\begin{abstract}
Bimetal composites have wide applications due to their excellent overall performance and relatively low comprehensive cost. The aim of this study is to investigate the forming behaviour of stainless/carbon steel bimetal composite during stamping by finite element method (FEM). In this work, the bonding interface of bimetal composite sheet was assumed to be perfect without delamination during the plastic forming process for simplicity. Uniaxial tensile tests on base metal (carbon steel) and compositing metal (stainless steel) were first carried out, respectively, in order to obtain the tensile properties of each of the component materials required in the forming simulation. Processing variables, including the layer stacking sequence, relative thickness ratios of two layers and friction were considered, and their effects on the distributions of circumferential stress and thickness strain were analysed. The bimetal composite sheet was set as the eight-node solid elements in the developed FEM model, which is effective for evaluating the distributions of circumferential stress and thickness strain, and predicting the high-risk region of necking during the stamping of bimetal composites. The simulation results can be used as an evaluation indicator of the capability of forming machine to ensure the bimetal composite can be safely formed.
\end{abstract}

\section{Introduction}

With the increasing demands for the multi-functionality of industrial products, many research efforts in materials science and engineering have focused on developing engineering materials of better mechanical capabilities and low comprehensive cost, such as bimetal composites [1]. Some common fabrication processes, such as hemming, bending, and large plastic forming, are adopted to further manufacture those bimetal and laminated metal composites [2]. In this case, many research works have been studied on the behaviours of bimetal composite sheets during the forming process.

Kapinski [3] presented the initial concepts of deep drawing process for bimetal elements of sheet materials. The inertia forces, plastic hardening behaviour of deformed material and dynamic stress were utilised in the problems of plasticity, and the experimental results were

\footnotetext{
"Corresponding author: jiang@uow.edu.au
} 
provided as well. Parsa et al. [4] studied the behaviour of two-layer aluminium-stainless steel laminated sheets during the direct drawing and reverse redrawing processes, i.e. the first and second drawing stages, by the finite element analysis and the experimental approach. Their studies indicated that the contact of stainless steel with the punch leads to the maximum drawing ratio in direct redrawing, but the aluminium should contact the punch in order to obtain the highest drawing ratio in reverse redrawing. Morovvati et al. [5] used the experimental and finite element investigation on wrinkling of circular two-layer metal sheets in the deep drawing process, and the influences of different blank holder forces on winkling were analysed. They also studied the relationship between the required forming force and the drawing radio. Likewise, Atrian et al. [6] studied the effects of different parameters, such as lubrication, blank-holder force and the diameter of the composite blank, on the deep drawing of steel/brass laminated sheets. However, in the previous works, those studied bimetal or laminated metal composites were thin sheets, but less people paid attention to the formability of the medium and thick bimetal composite.

In this study, the FEM investigation was conducted for the forming behaviour of the medium and thick bimetal sheets without the blank holder. The effects of some processing variables, including the layer stacking sequence, relative thickness ratios of two layers and friction on the forming force and the material flow were investigated and discussed. In order to predict the high-risk region of necking during the stamping process, the distributions of circumferential stress and thickness strain in the bimetal sheets were determined using the FE simulation.

\section{Tensile properties of bimetal composite}

A bimetal composite sheet, consisting of the duplex ferritic-austenitic stainless steel (2205) layer with the thickness of $3 \mathrm{~mm}$ and the carbon steel (AH36) layer with the thickness of $8 \mathrm{~mm}$, was bonded by the hot rolling as schematically shown in Fig. 1a. Chemical compositions of two essential components are shown in Table1. After roll bonding, the bonding interface was assumed to be thin and perfect without the delamination during the stamping process for simplicity in this work, and the morphology of bonding zone is shown in Fig. 1b.

Table 1. Chemical compositions of parent and clad metal plates.

\begin{tabular}{|l|l|l|l|l|l|l|l|l|}
\hline \multirow{2}{*}{ Steel } & \multicolumn{6}{|l|}{ Element (wt \%) } \\
\cline { 2 - 9 } & $\mathrm{C}$ & $\mathrm{Si}$ & $\mathrm{Mn}$ & $\mathrm{P}$ & $\mathrm{S}$ & $\mathrm{Cr}$ & $\mathrm{Mo}$ & $\mathrm{Ni}$ \\
\hline 2205 & 0.03 & 1.00 & 2.00 & 0.03 & 0.02 & $22-23$ & $3.0-3.5$ & $4.5-6.5$ \\
\hline AH36 & 0.18 & 0.50 & $0.90-1.60$ & 0.035 & 0.035 & 0.20 & 0.08 & 0.40 \\
\hline
\end{tabular}
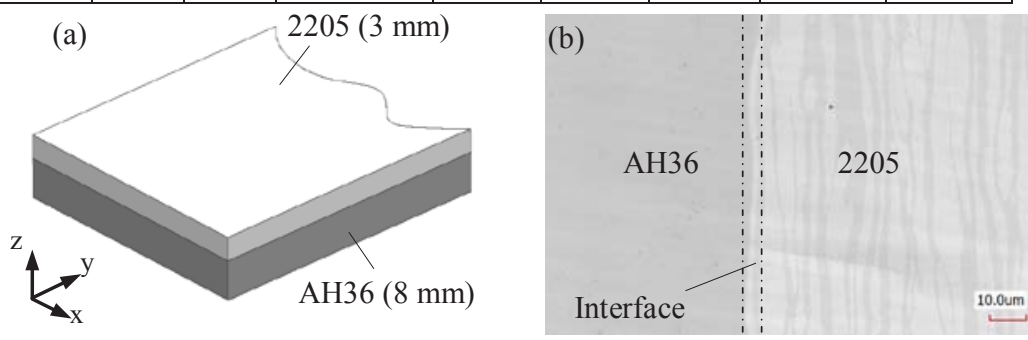

Fig. 1. Schematic illustration of (a) bimetal composite sheet, and (b) morphology of bonding zone.

Generally, the numerical simulation of stamping of the bimetal composite sheet can be conducted if the mechanical properties of its individual component layers and their 
thickness values were obtained. Therefore, the tensile tests on individual metal layer cut from the bimetal composite sheet by Spark Erosion Cutting (SEC) were carried out first, in order to obtain their individual mechanical properties. All uniaxial tensile tests were conducted on a universal testing machine Instron-8801 with crosshead speed of 0.5 $\mathrm{mm} / \mathrm{min}$, and the measurement of longitudinal deformation was carried out using an extensometer of gauge length $50 \mathrm{~mm}$. The results of tensile tests were shown in Fig. 2, which indicated experimental stress-strain curves for the bimetal plates with the relative thickness ratio 3:8, the 2205 stainless steel layer and the AH36 carbon steel layer. It is to note that the strength of these layers in the bimetal sheet was much different.

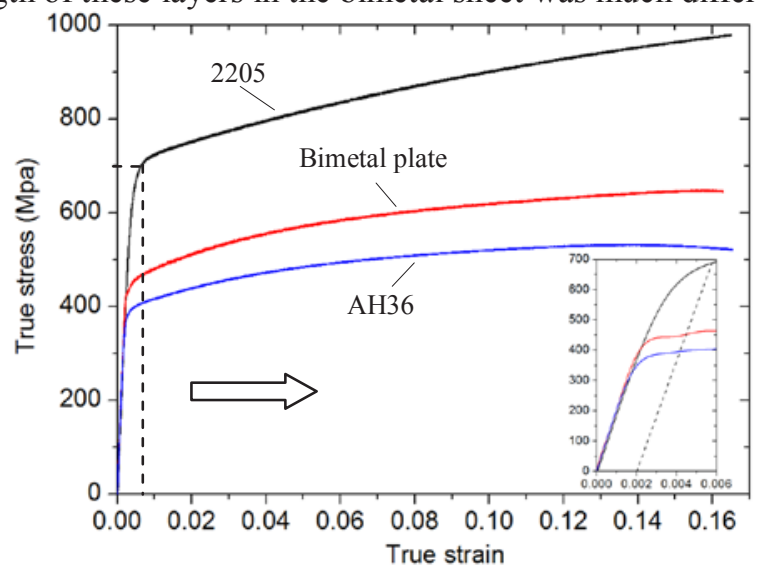

Fig. 2. Tensile properties of bimetal composite and its component layers.

\section{FE simulation}

The stamping was performed under two types of the sheet stacking sequence, i.e. the relative position of two layers, namely SC and CS. In the SC situation, the 2205 stainless steel layer was in contact with the punch and the AH36 carbon steel layer was positioned underneath. In the CS case, it was a reverse situation, i.e. the carbon steel of the bimetal sheet was in contact with the punch. The stamping die and the experimental procedures were illustrated in Fig. 3, and the dimensions of the major parts of the die set were shown in Table 2 as well.

Table 2. Dimensions of parts in the stamping die.

\begin{tabular}{|l|c|}
\hline Parameters & Dimensions (mm) \\
\hline Punch diameter & 138 \\
\hline Punch profile radius $^{1}$ & 14.5 \\
\hline Punch profile radius $^{2}$ & 24.5 \\
\hline Die diameter $^{1}$ & 160 \\
\hline Die profile radius $^{1}$ & 14 \\
\hline Die profile radius $^{2}$ & 25 \\
\hline Clearance between punch and die $^{2}$ & 11 \\
\hline
\end{tabular}




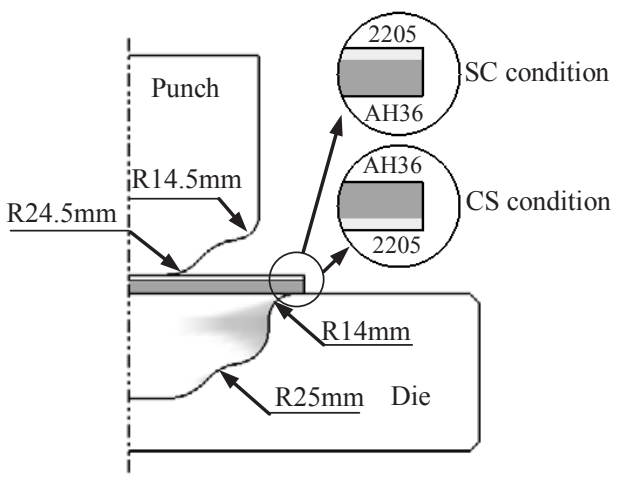

Fig. 3. Forming die set and experimental procedure.

The behaviour of the stamping of bimetal composite plate was more complicated than that of monolithic metal sheets, due to the mechanical properties of its component layers about the plastic yield stress and Young's moduli, relative thickness ratios of each layer and sheet stacking situations. Therefore, the FE simulation can be introduced to determine the situation of bimetal sheets stamping using the commercial software ABAQUS. The initial diameter of sheet model is $177 \mathrm{~mm}$ calculated by an inverse technique in the forming simulation software AUTOFORM, and the press speed of punch is $3.25 \mathrm{~mm} / \mathrm{s}$ assuming the rate independent behaviour without considerable discrepancies. In addition, the punch stroke is $65 \mathrm{~mm}$ according to the initial distance between the punch and die. As the bonding interface was assumed perfect, the bonding interface was set by a coherent node connection, and the model of composite plate was built together with different material properties in two layers obtained from previous tensile tests. The material was set as homogeneous and rate-independent following the von Mises yield criterion. An eight-node linear solid element C3D8R was used to mesh the plates, and there are 13824 elements in the FE model of bimetal plate, as shown in Fig. 4. In addition, the punch and die were set as a rigid body to reduce the computational time, and simulation conditions are shown in Table 3.

Table 3. FE simulation conditions.

\begin{tabular}{|l|c|}
\hline Items & Parameters \\
\hline Initial sheet diameter & $177 \mathrm{~mm}$ \\
\hline Punch speed & $3.25 \mathrm{~mm} / \mathrm{s}$ \\
\hline Punch stroke & $65 \mathrm{~mm}$ \\
\hline Element type & C3D $8 \mathrm{R}$ \\
\hline Element quantity & 13824 \\
\hline
\end{tabular}




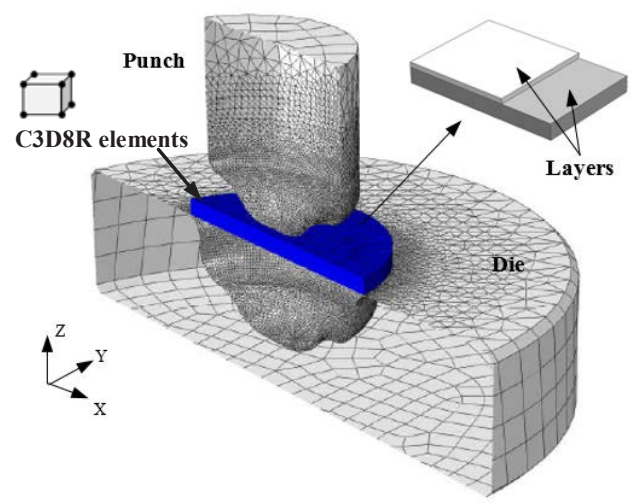

Fig. 4. FE model of bimetal plates stamping process.

\section{Results and discussions}

The effects of several conditions, such as the stacking sequence, relative thickness ratios and friction conditions, in the stamping process were presented and discussed. First, the layer stacking sequence and relative thickness ratios of two layers are of particular interest for the stamping behaviour of bimetal plates. To study the different influences of the plate stacking sequence and relative thickness ratios, four different simulations were preformed, i.e. the SC condition with the thickness ratios 3:8 and 1:1(total $11 \mathrm{~mm}$ thick), and CS condition with the thickness ratios 8:3 and 1:1. The load-stamping stroke curves were obtained from four different conditions under the dry condition with the frictional coefficient of 0.29 between tools and stamping plates, as shown in Fig. 5. There are three stages in the stamping process, i.e. the range of 0-40 $\mathrm{mm}$ (I stage), 40-60 $\mathrm{mm}$ (II stage) and $>60 \mathrm{~mm}$ (III stage). It is noted that the load values of four different conditions were almost the same in the initial I stage, but the difference began to appear and increased in the II stage. The first change position of load for all conditions appeared when the small profile corner of the punch (point A shown in Fig. 5) contacted the deformed plates, and the second change point of force appeared when the bigger profile corner of the die (point $\mathrm{C}$ shown in Fig. 5) contacted the deformed plates.

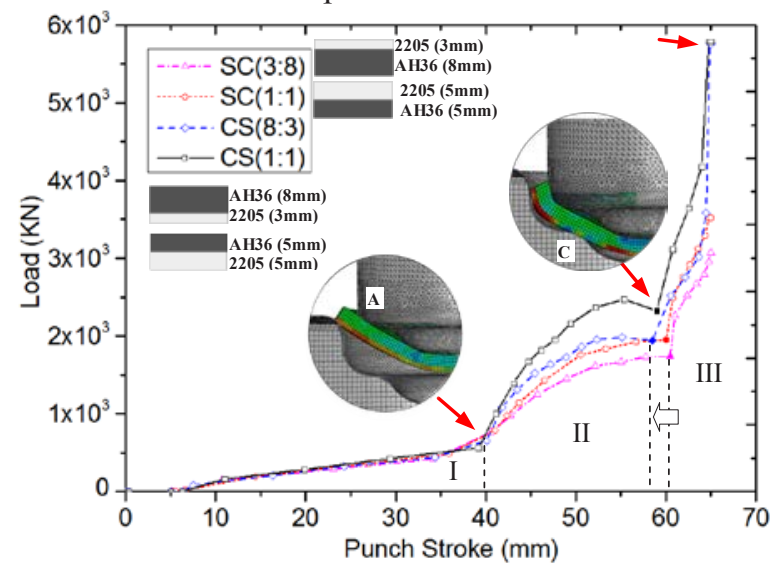

Fig. 5. Load-punch stroke curves in four different conditions.

It can be seen from the stage II of Fig. 5 that the forces of CS conditions all were larger than those of SC conditions with the same ratios of thickness and the former maximum 
forces were almost twice larger than that of the latter. Under the same stacking condition, the load increased with increasing the proportion of stainless steel thickness, i.e. the force of CS (1:1) condition was larger than that of CS (8:3) condition and this trend was the same for two SC conditions. In addition, it is worth noting that the beginning time of the stage III of stamping process in SC conditions was behand the time of CS conditions. The reasons were that the low carbon steel (AH36) with better plasticity was positioned in the underneath layer at large deformations resulting in the larger thickness reduction of the plate.

The friction condition between tools and stamping plates can affect the values of force and the formability of plates. Therefore, in order to observe the influence of friction, the load and stamping displacement curves in the SC condition with two previous thickness ratios were shown in Fig. 6, when the frictional coefficients were assumed to be $\mu=0.1$ and 0.29 under lubricant and dry conditions, respectively [6]. Fig. 6 indicates that the friction coefficient did not affect the load in the initial stage I of stamping process, because the contact area was small and the contact pressure was not sufficient at this stage. However, the load decreased in the stage II for both two different ratios with a smaller friction coefficient. The effect of the friction generally can be observed apparently at large deformations in the stamping process [7].

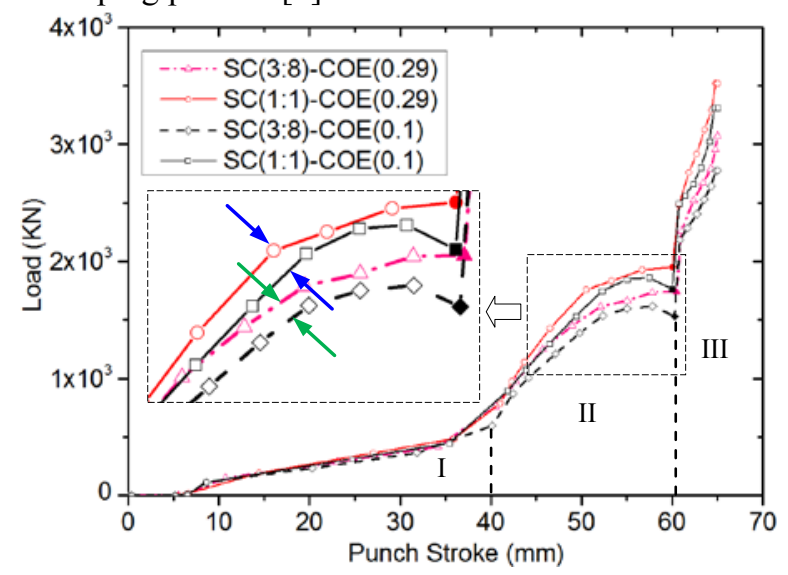

Fig. 6. Load-punch stroke curves with different friction coefficients

In addition to the load, the evaluation of material flow for bimetal plates stamping is important to reduce the defect or failure of forming products. The study of circumferential stress and thickness strain of the deformed component can determine the material flow situation and predict the occurrence of possible defects or even fracture $[8,9]$. There are two areas of profile radius in the punch (A and B corners shown in Fig. 7a) and one area of profile radius ( $\mathrm{C}$ corner shown in Fig. 7a), and the deformed plate was subjected to higher circumferential stress in some transition regions between those corners, which may cause the fracture in adjacent region. It is also shown in Fig. $7 \mathrm{~b}$ that the circumferential stress distribution of SC condition (3:8) and $\mu=0.1$ was zero for inside and outside layers near these areas. This trend was similar in other conditions. 


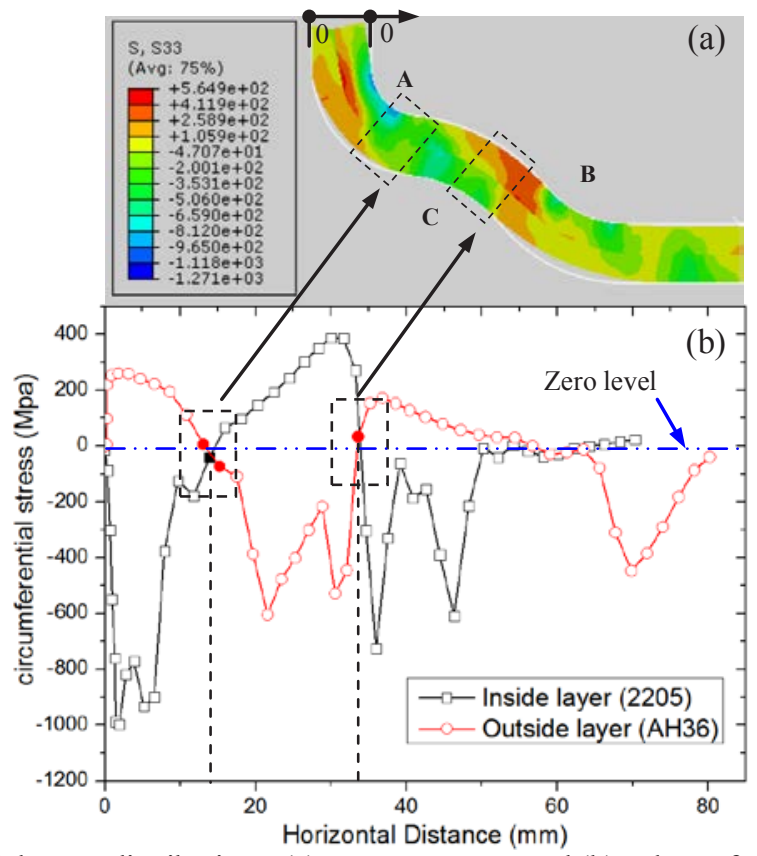

Fig. 7. Circumferential stress distributions: (a) stress contours, and (b) values of two layers.

Thickness strain values of both layers and total plates were also obtained from FE results. These values were determined in the thickness direction by measuring the thickness change of various regions from the centre of deformed component. Total and individual thickness strain distributions of SC and two friction conditions with the same thickness ratio (3:8) were shown in Fig. 8. It is shown that the small friction coefficients in comparison to dry condition made the component thickness to be uniform, i.e. the small total thickness reduction occurred, but the effect was not sufficient. The thickness of inner layer (2205) in both conditions fluctuated slightly from the centre to the edge of A corner of the punch, but increased by almost $15 \%$ in the A corner region due to the compression situation. In contrast, the thickness strain of outer layer (AH36) reached its low values in the $\mathrm{A}$ and $\mathrm{B}$ corner regions, and thus the same situation was for the total thickness strain. It was found that a lower thickness strain occurred in the underneath layer at a higher risk to fail. In addition, the negative thickness strain of total and individual layers at the centre of component was caused by the biaxial tensile stress state at this region [6].

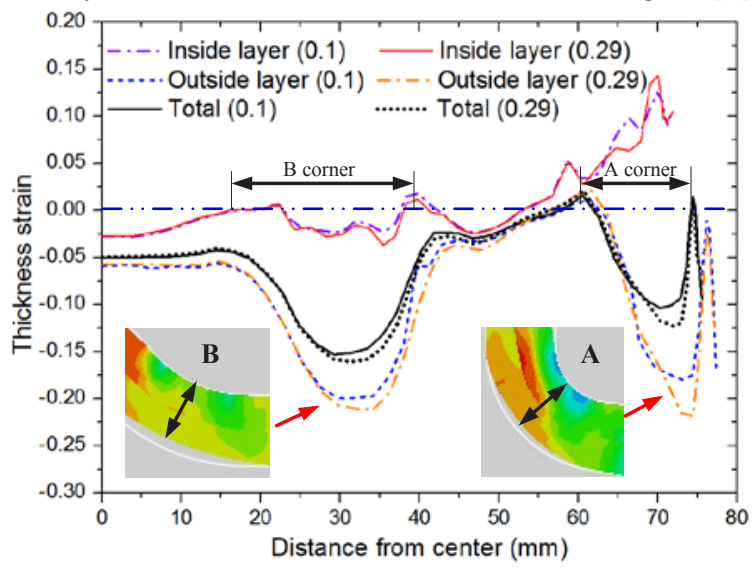

Fig. 8. Thickness strain distributions with different friction conditions. 


\section{Conclusions}

Forming characteristics of 2205 stainless/AH36 carbon steel bimetal composite during stamping were investigated based on the FE numerical simulation. The following conclusions can be obtained:

(1) The stacking sequence of layers has an influence on the load of punch, which means that the load is higher when the strong material (2205) is positioned in the underneath layer, but its effect is rather small in the initial stamping process.

(2) It has been found that, in all conditions, the punch load increases with the increase of the proportion of 2205 stainless steel thickness. Similarly, the influence is not sufficient at the beginning of the plastic deformed stage of bimetal plates.

(3) Friction coefficients affect the load in the second stage of stamping process in SC conditions, and the load decreases for different thickness ratios with a smaller friction coefficient.

(4) Distributions of the circumferential stress and thickness strain near the punch arears of profile radius indicate that the high risk of fracture appeared in these areas.

The authors wish to acknowledge the financial support from Baosteel-Australia Joint Research and Development Centre (BAJC) under project of BA-16009. The first author is greatly thankful for the scholarship support (IPTA) from University of Wollongong and scholarship support from China Scholarship Council (CSC).

\section{References}

1. K. Yilamu, et al., J Mater Process Tech, 210, 272-278 (2010)

2. B.X. Liu, et al., Mater Sci Eng: A, 679, 172-182 (2017)

3. S. Kapinski, J Mater Process Tech, 60, 197-200 (1996)

4. K.Y. Mohammad Habibi Parsaa, Norio Takakura, Int J Mech Sci, 43, 2331-2347 (2001)

5. M.R. Morovvati, Fatemi, A., and Sadighi, M., Int J Adva Manu Tech, 54, 113-121 (2011)

6. A. Atrian and Fereshteh-Saniee, F., Compos B: Eng, 47, 75-81 (2013)

7. F. Fereshteh-Saniee, Pillinger, I., and Hartley, P., J Mater Process Tech, 153, 151-156 (2004)

8. F. Dehghani and Salimi, M., In J Adva Manu Tech, 82, 163-177 (2016)

9. J.G. Liu and Xue, W., T Nonferr Metal Soc, 23, 964-969 (2013) 